\title{
Selective Inhibition of Tyrosine Kinase 2 With Deucravacitinib (BMS-986165) Compared With
}

Janus Kinase 1-3 Inhibitors

Anjaneya Chimalakonda, James Burke, ${ }^{*}$ Lihong Cheng, Ian Catlett, Aditya Patel, Jun Shen, Ihab G. Girgis, Subhashis Banerjee, John Throup Bristol Myers Squibb, Princeton, NJ, USA

*Employee at the time the analysis was conducted.

\section{Background}

Tyrosine kinase 2 (TYK2), an intracellular kinase involved in the pathogenesis of immune-mediated
inflammatory diseases (IMIDS), regulates signaling and functional responses downstream of the

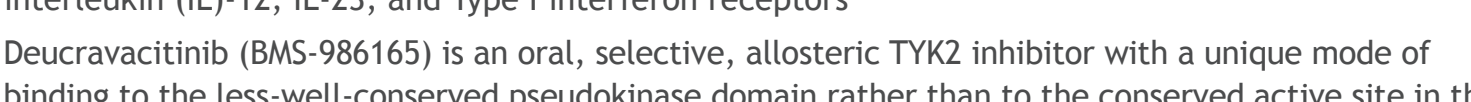

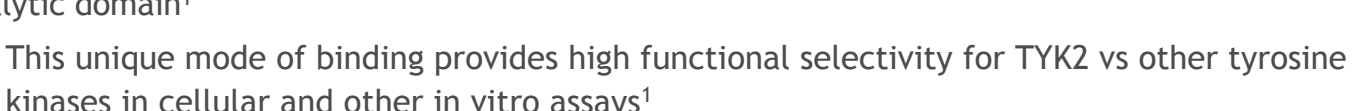
Allosteric inhibition may provide robust efficacy and a differentiated safety proffile vs other

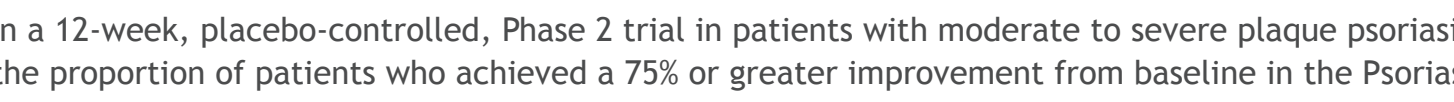
(1) placebo $77 \%$; $P<0.001)$. 2 Deucravacitinibi also had a favorable safety profile including no significan Deucravacitinibi is ucrentyly being evaluated in multiple IMIDs including plaque psoriasis, psoriatic
arthritis, inflammatory bowel disease, and lupus Objective To compare the selectivity of deucravacitinin vs the approved Janus kinase (JAK) inhibitors
tofacitinii, upadacitinit, and baricitinii, at clinically relevant doses and plasma concentrations Methods

In vitro whole blood assims
developed (Table 1)

mined using these assays, as well as thill coefficieints for inhibitition

1. In vitro whole blood assays for JAK 1-3 and TYK2 inhibitors



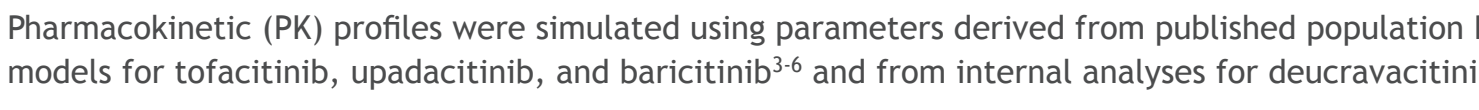

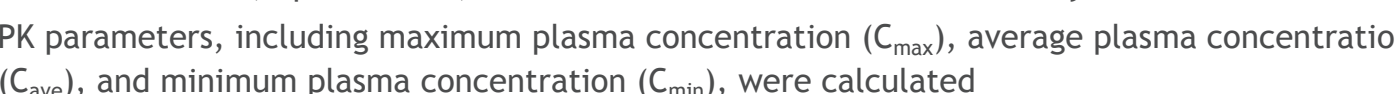

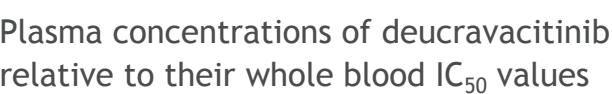

政 - It the whole blood I I S value was $h$, Additionally, key exposure parameters $C$
their individual whole blood ICs values

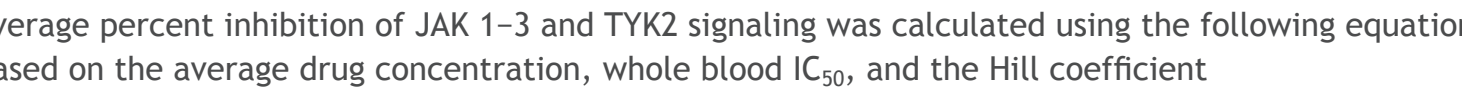
Percent intibition $=100 /\left(1+\left[\left(\left[1 S_{0} / X\right)^{-} H\right]\right)\right.$ where $X$ is the average drug concentration and $H$ is the
Hill coefficient

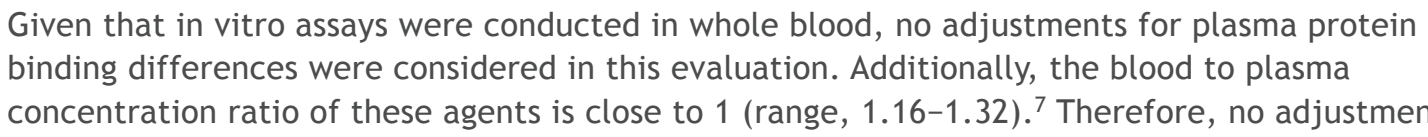
concentration ras
were considered

\section{Results}

In vitro whole blood $\mathrm{IC}_{50}$

Based on in vitro whole blood $1 C$
compared With JAK $1 / 3$ or $J A K 2$

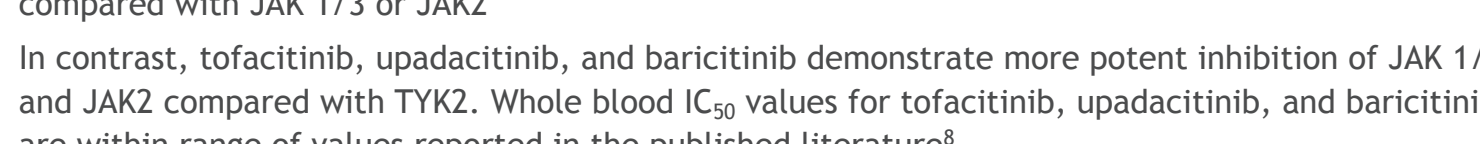

Table 2. In vitro whole blood IC $\mathrm{S}_{5}$ values for JAK 1-3 and TYK2 inhibitors

\begin{tabular}{|c|c|c|c|c|}
\hline & \multicolumn{4}{|c|}{ Whole blood $\mathrm{I}_{50}(95 \% \% \mathrm{C}), \mathrm{nM}$} \\
\hline Signaling kinase readout & Tofactitinib & Baricitinib & Upadacitinib & Deucravacitinit \\
\hline JAK 1/3 (IL-2-induced PSTAT5) & $\begin{array}{l}17 \\
(15-19)\end{array}$ & $(8.7113)$ & $\begin{array}{l}7.8 \\
(6.5-9.5)\end{array}$ & $\begin{array}{l}14646 \\
(146-1872)\end{array}$ \\
\hline JAK2 (TPO-induced PSTAT3) & ${ }_{(182-258)}^{21}$ & $\begin{array}{c}32 \\
(28-36)\end{array}$ & $\begin{array}{l}41 \\
(36-47)\end{array}$ & 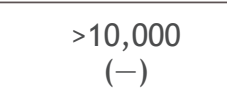 \\
\hline TTK2 (LL-12-induced IFN-r release & $\begin{array}{lll}5059 \\
(3767-7026)\end{array}$ & $\begin{array}{l}23551 \\
(1834-2980)\end{array}$ & $\begin{array}{l}3385 \\
(2346-6208)\end{array}$ & $\left.\begin{array}{l}40 \\
(29-55)\end{array}\right)$ \\
\hline
\end{tabular}

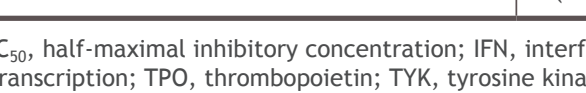

Daily percent inhibition by JAK 1-3 and TYK2 inhibitors

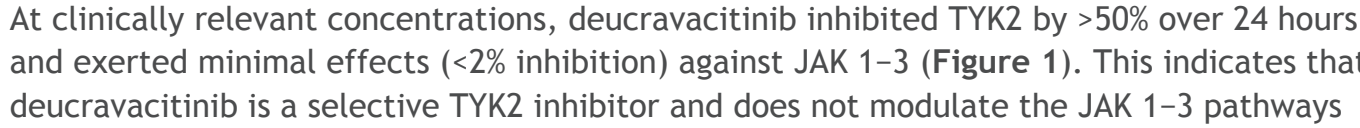

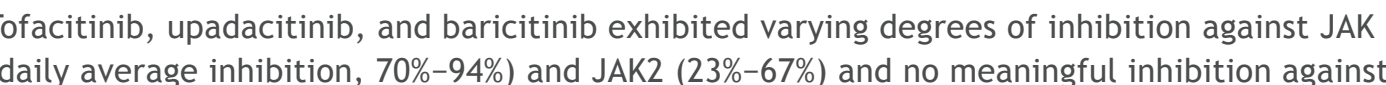

Figure 1. Daily percent inhibition by JAK 1-3 and TYK2 inhibitors

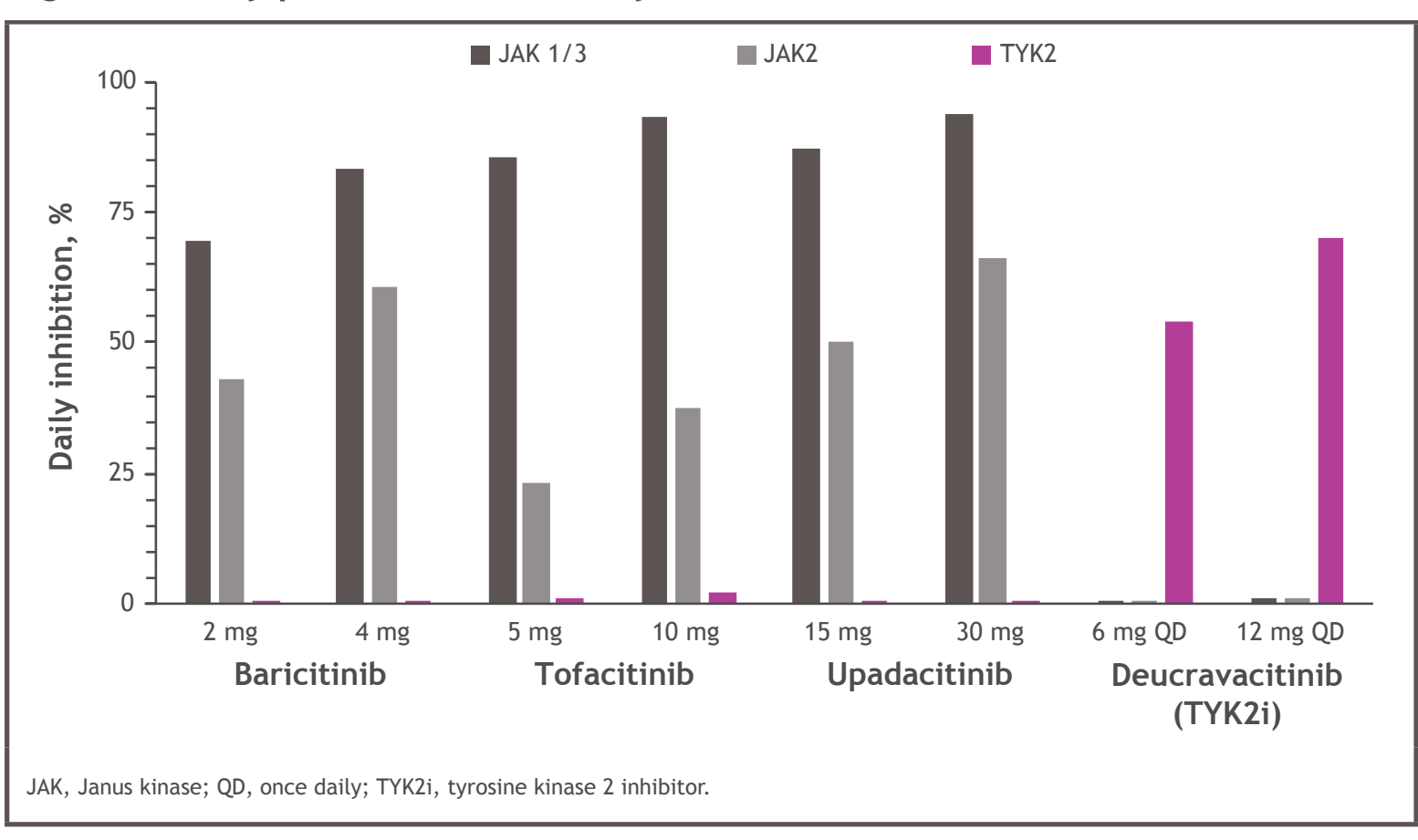

JAK 1-3 and TYK2 inhibitor plasma concentrations and whole blood $\mathrm{IC}_{50}$

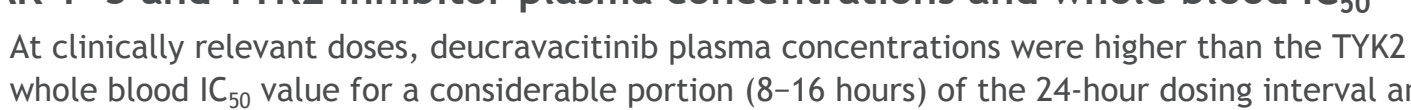

considerably lower than the JAK $1-3 K_{50}$ values throughout the dosing interval (Figure 2$)$

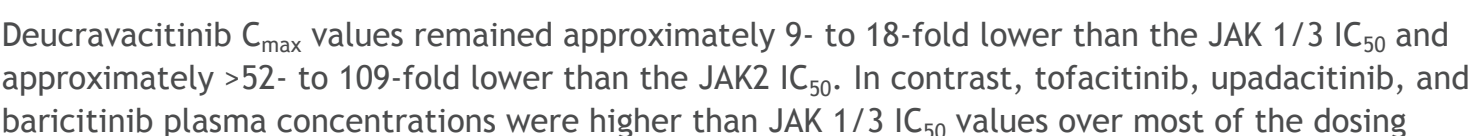
baricitinib plasma concentrations were higher than $J A K 1 / 3 I_{50}$ values over most of the dosing

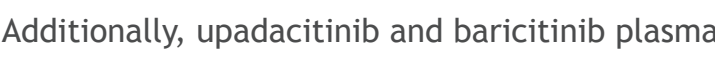

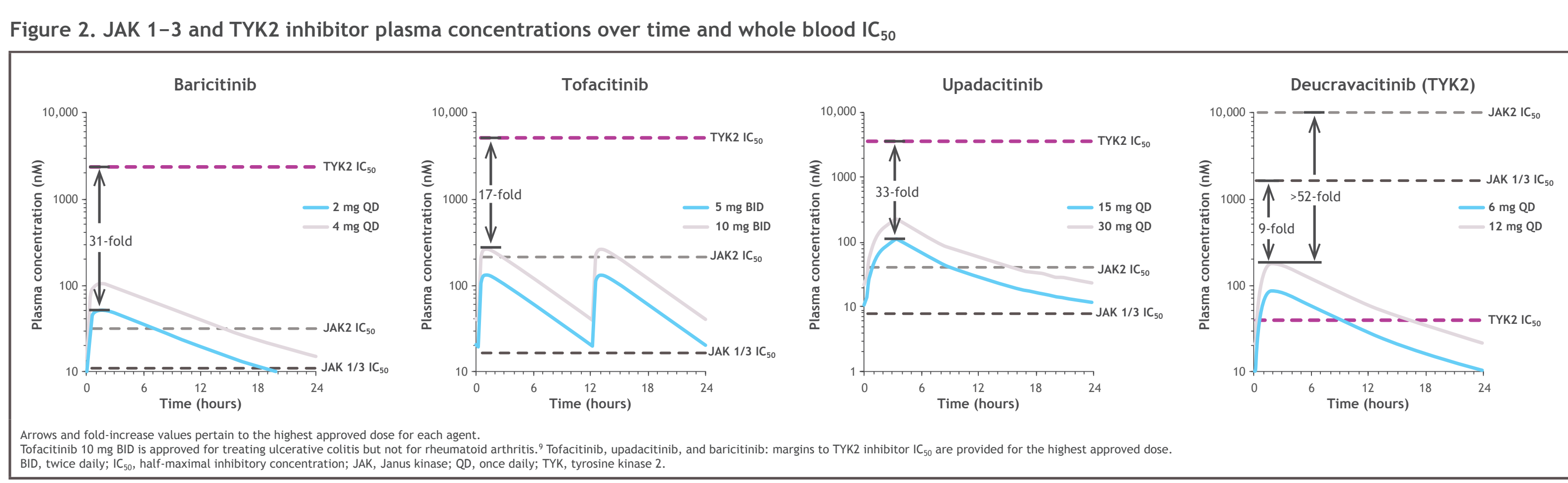



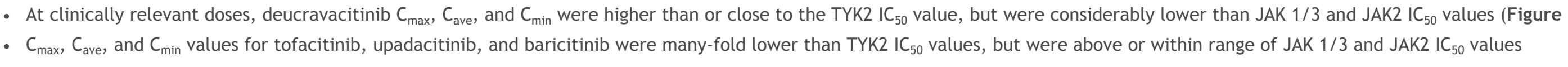
Figure 3. JAK 1-3 and TYK2 inhibitor pharmacokinetic parameters and whole blood IC

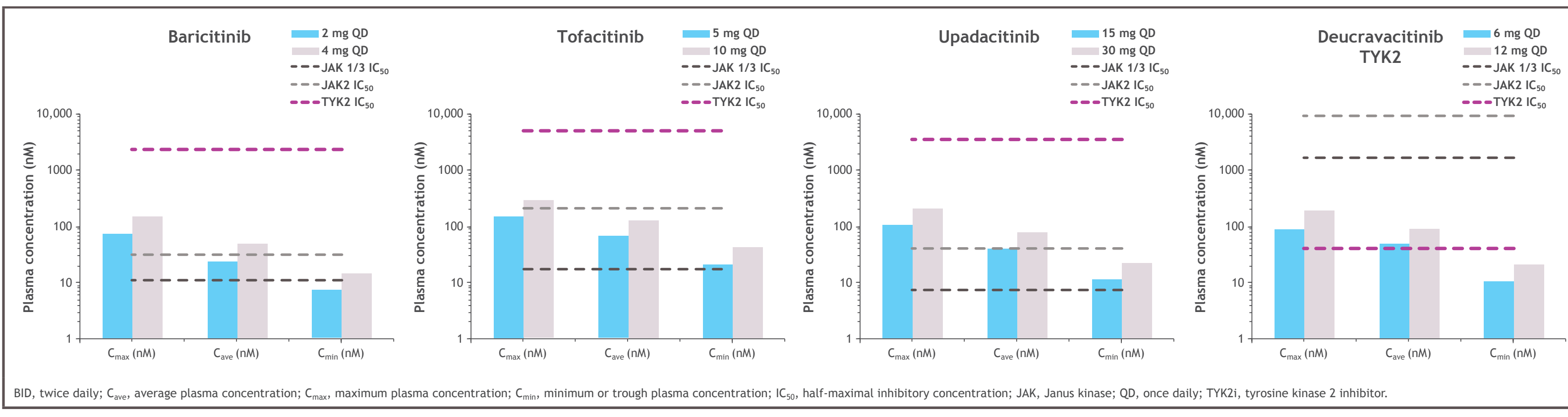

\section{Conclusions}

- This analysis confirms that deucravacitinini is a highly selective, allosteric TKK2 inhibitor with
minimal or no activity against $\mathrm{AAK} 1-3$ - Selective TKK2 inhibition is consistent with the reduced potential for treatment-related

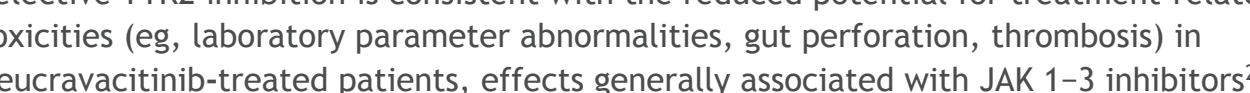

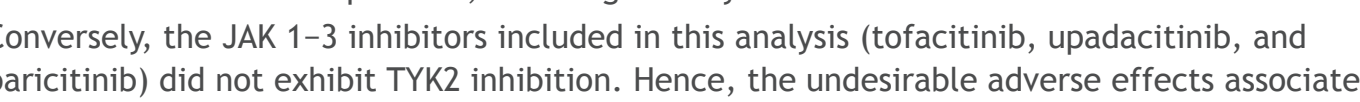

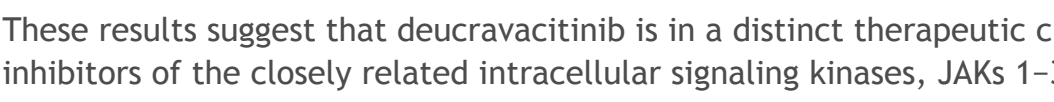



References



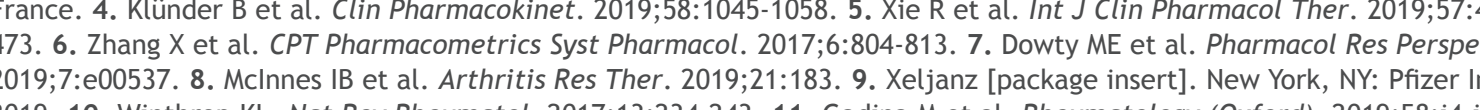
Acknowledgments

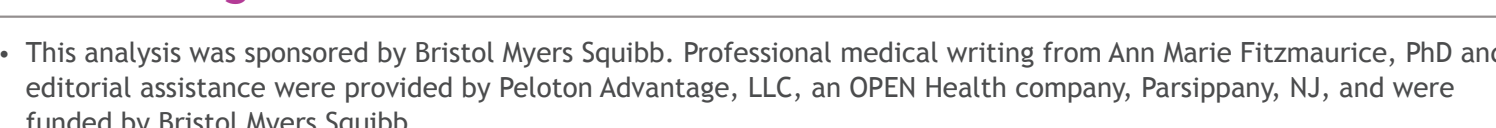

Relationships and Activities

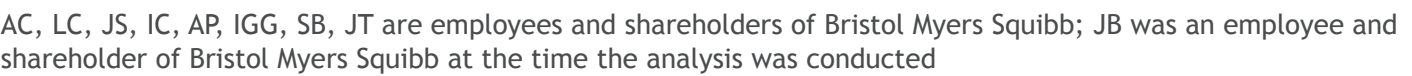

